\title{
RECHERCHES SUR LE DÉVELOPPEMENT ET L'IDENIIFICATION DE PLAGIORCHIS (MULTIGLANDULARIS) CIRRATUS (Rudolphi 1802)
}

\section{Etude du cycle évolutif}

\section{Par Alice BUTTNER et Cécile VACHER}

Depuis de nombreuses années, au lieudit « Moulin de Thuet», un certain pourcentage de Gammarus pulex L. récoltés dans la Veude, non loin du Domaine Universitaire de Richelieu (Indre-etLoire), présentent des métacercaires sous-cuticulaires de type Plagiorchis.

L'ingestion de ces métacercaires par des souris d'élevage a permis d'obtenir un grand nombre d'exemplaires adultes, dont certains ont été soumis, à fin de détermination, à R.-Ph. Dollfus. Cet auteur donne ci-après une description détaillée et une discussion sur la position systématique de ces Trématodes qu'il identifie à l'espèce Plagiorchis (Multiglandularis) cirratus (Rudolphi, 1802).

Cependant, ces Distomes assez communs chez de nombreux Oiseaux d'Europe et d'Asie Occidentale, n'ont jamais été trouvés dans les autopsies d'Oiseaux pratiquées chaque été, depuis près de 30 ans, à la Station expérimentale de Parasitologie de Richelieu, ce qui préjugerait peut-être en faveur d'un hôte migrateur, de passage en une autre saison au voisinage des gìtes parasités.

En revanche, il nous a été relativement facile de dépister les hôtes naturels hébergeant les premiers stades larvaires de cet Helminthe, et nous rapportons ici les résultats de nos recherches concernant le cycle évolutif de ce Plagiorchiidx.

\section{\$* \\ Matériel et technignues}

Le matériel vivant que nous avons utilisé (Mollusques, Gammares, Aselles, Poissons, Crapauds, Grenouilles, Couleuvres, Souris) a été prélevé, soit dans les gîtes infestés, soit dans les canaux du Domaine uni- 
versitaire de Richelieu, les ruisseaux du Bois de Boulogne à Paris, ou les élevages de nos laboratoires.

Les localisations habituelles des kystes métacercariens chez les Gammares ont été mises en évidence par examen des spécimens parasités, comprimés entre deux lames maintenues aux extrémités par des pinces robustes. La morphologie interne des métacercaires mùres a été étudiée par dissection des Gammares et libération des kystes dans l'eau physiologique, ou dans des produits à base d'alcool polyvinylique (rhodoviol H.S. 10 ou H.S. 100 en solution saturée dans l'eau distillée), et légèrement additionnés de rouge neutre ou de vert de méthyle. Après incision de la paroi kystique, la métacercaire dégagée de son enveloppe a été dessinée à frais, à la chambre claire, ou colorée au carmin chlorhydrique (1).

Les sporocystes et xiphidiocercaires ont été examinés vivants dans du rhodoviol H.S. 100 en solution, ou après fixation à l'alcool-formol et coloration au carmin chlorhydrique ou bleu de méthylène.

Les Distomes adultes ont été fixés à l'alcool chaud et colorés au carmin chlorhydrique, bleu de Unna-orcéine, bleu de Unna-tannin orange, bleu polychrome-orcéine et bleu polychrome-tannin orange.

\section{$* *$}

\section{Recherches sur le cycle évolutif}

La présence d'un stylet dans les kystes jeunes, l'enkystement des métacercaires chez des Gammares, apportaient déjà deux éléments d'information sur le cycle biologique de ce Trématode :

$1^{\circ}$ la cercaire est une xiphidiocercaire du groupe «armata » Lühe, 1909, caractéristique commune à tous les Plagiorchidés ;

$2^{\circ}$ le second hôte intermédiaire est un Arthropode aquatique.

\section{a) Les hôtes:}

Premier hòte intermédiaire. - Les Mollusques présents dans la Veude, dans le biotope des Gammares infestés, appartenaient aux espèces suivantes: Limnaea stagnalis (L.), L. limosa (L.), L. palustris (Müll.), Bithynia tentaculata (L.), Vivipara vivipara (L.), Theodoxia fluviatilis (L.), Pisidium amnicum (O. F. Müller).

(1) Des essais d'évolution de métacercaires mûres dans leurs kystes, sous la seule action de la température ont été tentés sans résultats : 17 métacercaires âgées de 20 jours, placées (non aseptiquement) dans l'eau physiologique, à une température variant de 30 ì $35^{\circ} \mathrm{C}$., ont subsisté pendant 10 jours sans manifestation visible d'une quelconque évolution. Des kystes témoins, placés dans les mêmes conditions, mais à la température du laboratoire $\left(22-25^{\circ} \mathrm{C}\right.$.), étaient déjà en voie de lyse au bout de 48 heures. Peut-être le maintien de kystes dans un milieu aseptique, à température élevée, permettrait-il un développement in vitro de la métacercaire en adulte. 
C'est chez Limnea stagnalis et L. limosa seulement que nous avons pu identifier les xiphidiocercaires s'enkystant chez les Gammares infestés. Les pourcentages d'infestation des Limnées étaient assez faibles (10 à $15 \%$ ), mais les émissions quotidiennes de cercaires étaient massives.

Deuxième hôte intermédiaire. - Mis en présence de ces cercaires, des Gammarus pulex L. d'élevage succombèrent en quelques heures, le corps, les antennes et les pattes criblés de cercaires enkystées. Une attraction intense concentrait les cercaires autour de la tête, du corps et des appendices, malgré les contractions multiples du Gammare pour se défendre contre cette attaque en masse. Les larves rampaient sur la cuticule, introduisaient leur stylet au point de jonction de deux articles et s'enfonçaient dans les téguments en abandonnant leur queue. L'enkystement se produisait en 30 à 60 minutes, et le stylet libéré flottait dans le kyste pendant deux ou trois jours. Les kystes grandissaient lentement et atteignaient leur maturité en 12 à 15 jours, la température ambiante oscillant autour de $25^{\circ} \mathrm{C}$.

Des Crustacés Isopodes (Asellus aquaticus L.), provenant de ruisseaux du Bois de Boulogne, à Paris, furent exposés à leur tour aux attaques de ces xiphidiocercaires. Au bout de 24 heures, ils avaient contracté des infestations aussi fortement positives que les Gammares. Il parait donc vraisemblable que d'autres Arthropodes aquatiques (larves d'Insectes par exemple) puissent avoir une réceptivité égale à ce parasitisme.

A l'inverse, des organismes appartenant à des groupes zoologiques différents, comme des têtards d'Alytes obstetricans (Laur.) en particulier, n'exerçaient aucune attirance sur les cercaires émises à leur contact et ne se sont pas infestés.

Hôte déFinitif. - A partir des kystes mûrs, obtenus expérimentalement chez des Gammares ou des Aselles, des infestations d'animaux pœeilothermes ou homœothermes ont été tentées, afin d'obtenir la forme adulte du parasite.

1) Infestation d'animaux pœcilorhermes : Ont été soumis à cette infestation, par ingestion de 200 à 300 kystes âgés de 15 à 20 jours, les animaux suivants: Cottus gobio, Natrix natrix, Natrix maura, Bufo vulgaris, Rana esculenta et Rana dalmatina.

Protocoles d'infestation :

Cottus gobio est sacrifié 12 jours après l'infestation. Autopsie négative. 
Natrix natrix est sacrifiée 7 jours après l'infestation. Autopsie négative.

Natrix maura est sacrifiée 11 jours après l'infestation. Autopsie négative.

Bufo vulgaris est sacrifiè 18 jours après l'infestation. Autopsie négative.

Rana esculenta est sacrifiée 5 jours après l'infestation. Autopsie négative.

R. dalmatina est sacrifiée 5 jours après l'infestation. Autopsie négative.

L'insuccès de ces tentatives d'évolution chez des Vertébrés à température variable nous incite à reprendre cette expérimentation avec des homœothermes.

2) Infestation de Souris blanches : Une première série d'expériences est effectuée sur trois Mus musculus albus, à partir de Gammarus pulex, infestés spontanément au gite de Thuet.

L'infestation des Souris s'opère assez facilement en dilacérant les Gammares et en mélangeant les débris à du biscuit pilé dans un verre de montre. Chaque Souris ingère ainsi en moyenne de dix à quinze kystes mûrs. L'examen quotidien des fèces nous permet de trouver, six jours après l'infestation, des œufs operculés en voie de segmentation, témoignant de la présence dans l'intestin de quelques Trématodes parvenus à l'état adulte.

La première autopsie est pratiquée sept jours après l'infestation. Elle permet de retrouver dans l'intestin moyen six spécimens adultes de Plagiorchis qui, ainsi qu'il est dit plus loin (1), appartiennent au sousgenre Multiglandularis et à l'espèce cirratus.

Une deuxième Souris est sacrifiée le onzième jour ; quatre nouveaux exemplaires adultes sont retirés de son intestin; le tube digestif de ces douves était rempli d'hématies et intensément coloré en rouge.

La troisième Souris est tuée le treizième jour, et huit Plagiorchis adultes sont récoltés dans son intestin grêle.

A partir du sixième jour d'infestation, des œufs sont observés journellement dans les selles, mais ils ne sont jamais embryonnés.

Une deuxième série d'infestations est effectuée sur trois Musculus albus à partir d'Asellus aquaticus expérimentalement parasités. L'infestation de ces Rongeurs est réalisée comme précédemment par la voie orale, mais avec un plus grand nombre de kystes, âgés de 13 jours. Chaque animal ingère de 30 à 70 métacercaires.

(1) Voir $\mathrm{II}^{e}$ partie de cet article : Description et Identification par R.-Ph. Dollfus, 
Les Souris sont sacrifiées respectivement les onzième, treizième et trente-deuxième jours; 64 vers adultes sont recueillis à la première autopsie ; 31 à la seconde, tandis qu'ils ont totalement disparu à la dernière.

L'examen quotidien des selles fait apparaître les premiers œufs le sixième jour. Ces œufs augmentent en nombre jusqu'au dixième jour environ, se maintiennent au maximum jusque vers le vingtième jour, pour décroître et disparaître (avec les derniers vers adultes) le trentième jour (1). Ces œufs n'étaient jamais embryonnés.

D'après ces expériences, la maturation de l'adulte chez la Souris

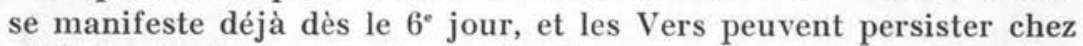
cet hôte pendant près d'un mois (2).

3) Maturation des œufs : Les œufs libérés dans l'intestin et expulsés avec les fèces ne sont pas embryonnés. A la température du laboratoire $\left(22-25^{\circ}\right.$ C. $)$, la division cellulaire se poursuit lentement, avec des diapauses, mais sans parvenir à son terme. Pour obtenir l'embryon vivant, il a fallu faire intervenir un Mollusque et lui faire ingérer les œufs en voie de division. N'ayant plus à notre disposition d'exemplaires des Limnées, hôtes intermédiaires, nous avons utilisé des Australorbis glabratus d'élevage, et le passage en transit dans le milieu intestinal de ces Planorbes a suffi pour réactiver et achever la segmentation de l'œuf.

\section{b) Les stades évolutifs :}

Sporocystes (fig. 1) : Les sporocystes fils, où se développent directement les cercaires, sont des éléments sacciformes, généralement blanchâtres, qui envahissent et détruisent progressivement la glande digestive du Mollusque. Ils mesurent en moyenne de 600 à $900 \mu$ de long sur 120 à $150 \mu$ de large, et sont fixés à l'hépato-pancréas par un pied granuleux de 150 à $200 \mu$ sur 70 à $100 \mu$. Ils possèdent une paroi cellulaire épaisse, formée de plusieurs couches cellulaires et d'une assise interne constituée par des cellules égales et aplaties.

Xiphidiocercaires (fig. 2): Les cercaires qui s'échappent des sporocystes ont achevé leur développement. Elles s'apparentent aux nombreuses cercaires du groupe «armata 》 décrites dans la litté-

(1) Cette courbe de production des œufs, qui accuse un maximum en plateau pendant 8 à 10 jours, présente des analogies avec celle décrite par J. Yamashita en 1953 pour Plagiorchis muris Tanabé, 1922.

(2) Une erreur dans les dates d'infestation de ces Souris nous a fait indiquer à tort, dans une publication antérieure (1959), que la longévité des vers chez la Souris pouvait atteindre 60 jours. 


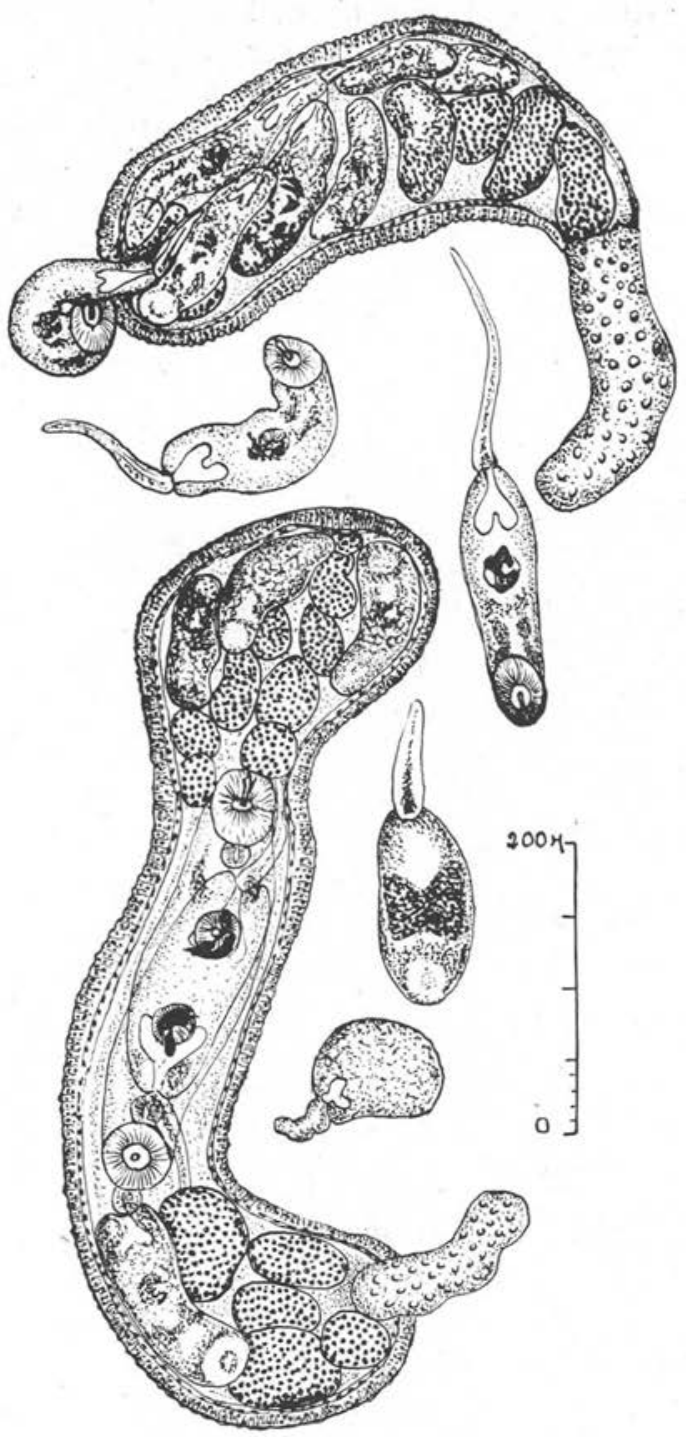

FIG. 1. - Plagiorchis cirratus : sporocystes ; cercaires mùres et immatures.

rature (Lühe, 1909 ; Cort, 1915 ; Sewell, 1922 ; Dụbois, 1929 ; Wesenberg-Lund, 1931), ou rapportées dans les traités (Bẻn Dawes, 1946 ; Yamaguti, 1958). Mais il ne semble pas qu'elles puissent s'identifier à une forme précédemment décrite. 
Fixées en extension par la chaleur, leurs dimensions moyennes sont, pour le corps, de 220 à $250 \mu$ de long sur 130 à $150 \mu$ de large ; pour l'appendice caudal, de 220 à $250 \mu$ de long sur 20 à $40 \mu$ de large. Ventouse orale : $60 \times 60 \mu$; court prépharynx ; pharynx : 19-20 $\times 19-20 \mu$; acetabulum : 30-35 $\times 35-40 \mu$; stylet : $28-30 \times$ 5-7 $\mu$. Des rangées parallèles d'épines disposées en quinconces
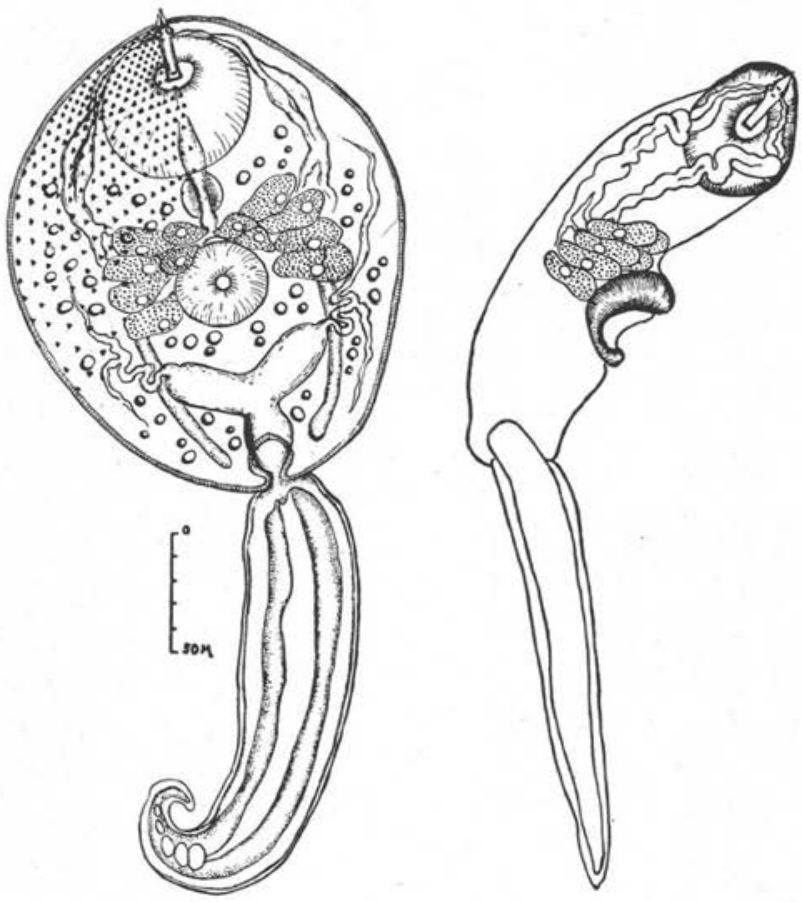

FIG. 2, $-P$. cirratus : Xiphiciiocercaire.

recouvrent la cuticule dans la moitié antérieure du corps et s'espacent graduellement jusqu'à la ventouse ventrale. Le corps est rempli de granules réfringents, rendant l'observation souvent difficile. L'œsophage se divise en avant de la ventouse ventrale en deux cæca atteignant l'extrémité postérieure. Les glandes céphaliques, au nombre de six paires, sont disposées de part et d'autre de l'acetabulum en deux groupes inégaux, l'un de deux cellules, submédian, l'autre de quatre cellules, plus externe. La vessie en $\mathrm{Y}$ est formée de trois branches relativement égales; un conduit d'excrétion traverse la queue dans toute sa longueur. Le système excréteur 

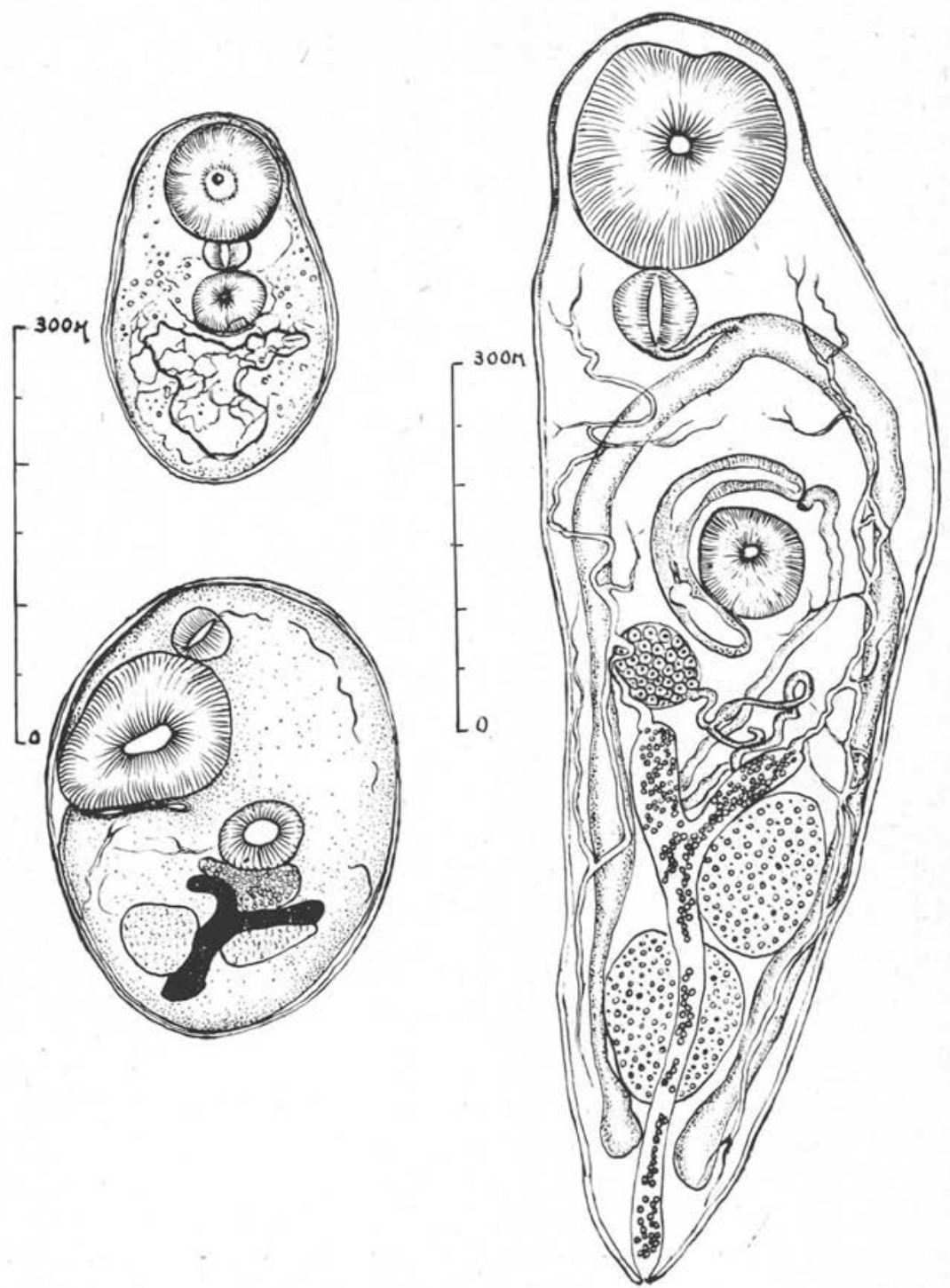

Fig. 3. - P. cirratus : Métacercaires; à gauche, kyste de 5 jours et kyste mûr avec ébauches génitales; à droite, métacercaire mûre extraite de son kyste.

est malaisé à mettre en évidence; nous ne l'avons observé qu'imparfaitement, mais il semble bien devoir être rapporté au type "polyadena " Cort 1919, dont la formule est :

$2[(3+3+3)+(3+3+3)]=36$ flammes vibratiles. 
Métacercaires (fig. 3) : Après enkystement de la cercaire, les glandes cystogènes se résorbent et les concrétions réfringentes vont s'accumuler dans la vessie. Stylet libre dans le kyste pendant les trois premiers jours. Kyste de cinq jours : 250-270 $\times 150-170 \mu$; kyste mùr : $300-350 \times 240-260 \mu$.

Extraite de son kyste, la métacercaire mesure 1.000 à $1.200 \times$ $300-350 \mu$; ventouse orale : $\pm 180 \times 180 \mu$; pharynx : $\pm 65 \times$

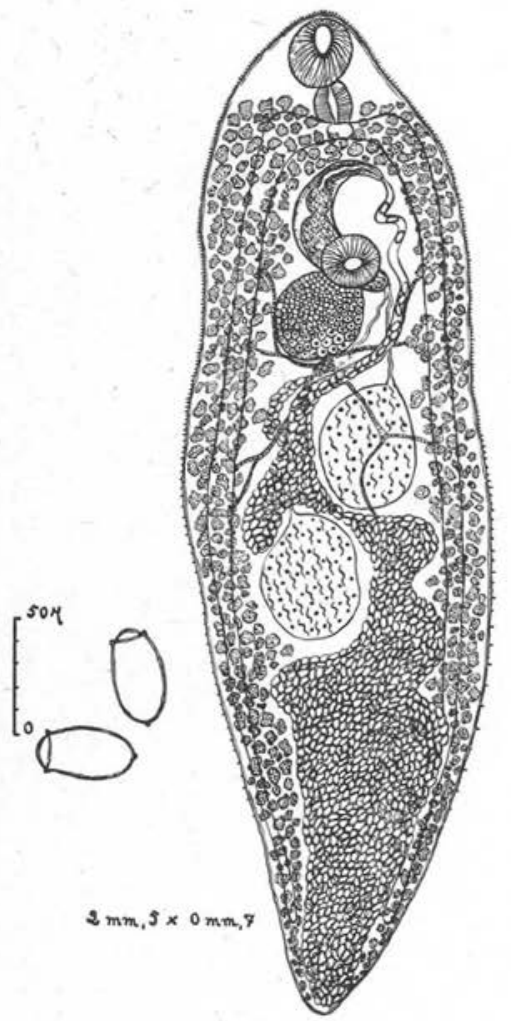

FIG. 4. - P. cirratus : Adulte. A gauche, forme et dimensions des æufs retrouvès dans les fèces, chez la souris blanche.

$65 \mu$; acetabulum : $\pm 90 \times 90 \mu$; œsophage : $90 \mu$ de long; cæca atteignant presque l'extrémité postérieure du corps; vessie en $\mathrm{Y}$ dont le tronc médian s'est considérablement allongé et se divise au niveau du milieu du testicule antérieur. Petit ovaire sphérique latéral, à droite et au-dessous de l'acetabulum ; canal de Laurer présent; deux testicules ovalaires, beaucoup plus développés que l'ovaire, en position oblique dans la moitié postérieure du corps, le testicule antérieur atteignant presque le sommet d'une des cornes 
latérales de la vessie, tandis que le bord distal du testicule postérieur se situe sensiblement au premier tiers de la vessie; poche du cirre très développée, en forme de $\mathrm{C}$, contenant une vésicule séminale double, le pénis et les glandes prostatiques, et entourant la ventouse ventrale. Pore génital situé juste au-dessus de l'acetabulum et légèrement sur la gauche.

Adultes (fig. 4) : La description en est donnée ci-après par R.-Ph. Dollfus. Mentionnons simplement les dimensions moyennes du corps : $2.500-2.800 \times 500-600 \mu$.

$$
* *
$$

\section{Discussion et conclusion}

Il nous a paru intéressant de comparer le cycle évolutif de ce Plagiorchis à ceux déjà décrits pour d'autres espèces rapportées au même genre, et dont les cercaires armées s'enkystent chez des Arthropodes aquatiques.

Nous rappelons, dans le tableau ci-après, les différents hôtes qui interviennent dans ces cycles biologiques, aussi bien pour les espèces identifiées au sous-genre Plagiorchis qu'au sous-genre Multiglandularis.

Le trait le plus frappant qui ressort de ce tableau, c'est l'ubiquité présentée à tous les stades évolutifs par ces Plagiorchis. On ne peut dégager de ces évolutions particulières un schéma général dans lequel viendraient s'intégrer les cycles évolutifs des espèces de l'un ou l'autre sous-genre.

Le premier hôte est le plus souvent un Gastropode Pulmoné; mais il peut être aussi un Gastropode Prosobranche [Bithynia tentaculata pour $P$. (M.) arcuatus].

Le second hôte est, dans tous les cas, un Arthropode aquatique ; mais on trouve parfois également les métacercaires chez les Mollusques premiers hôtes $[P$. (P.) proximus ; P. (M.) muris $]$.

Enfin, l'hôte définitif s'observe dans les groupes les plus variés de Vertébrés à sang chaud (Oiseaux, Mammifères) et, éventuellement, chez des animaux à température variable [Chamaeleon, Bufo, pour $P$. (P.) ramlianus $]$.

Cette disparité dans les cycles, chez des espèces aussi étroitement apparentées, révèle des facultés d'adaptation très diverses au cours de l'évolution. Or cette « indifférence » biologique est un phénomène assez inhabituel chez les Trématodes où la spécificité parasitaire, pendant les stades larvaires tout au moins, est en général très 


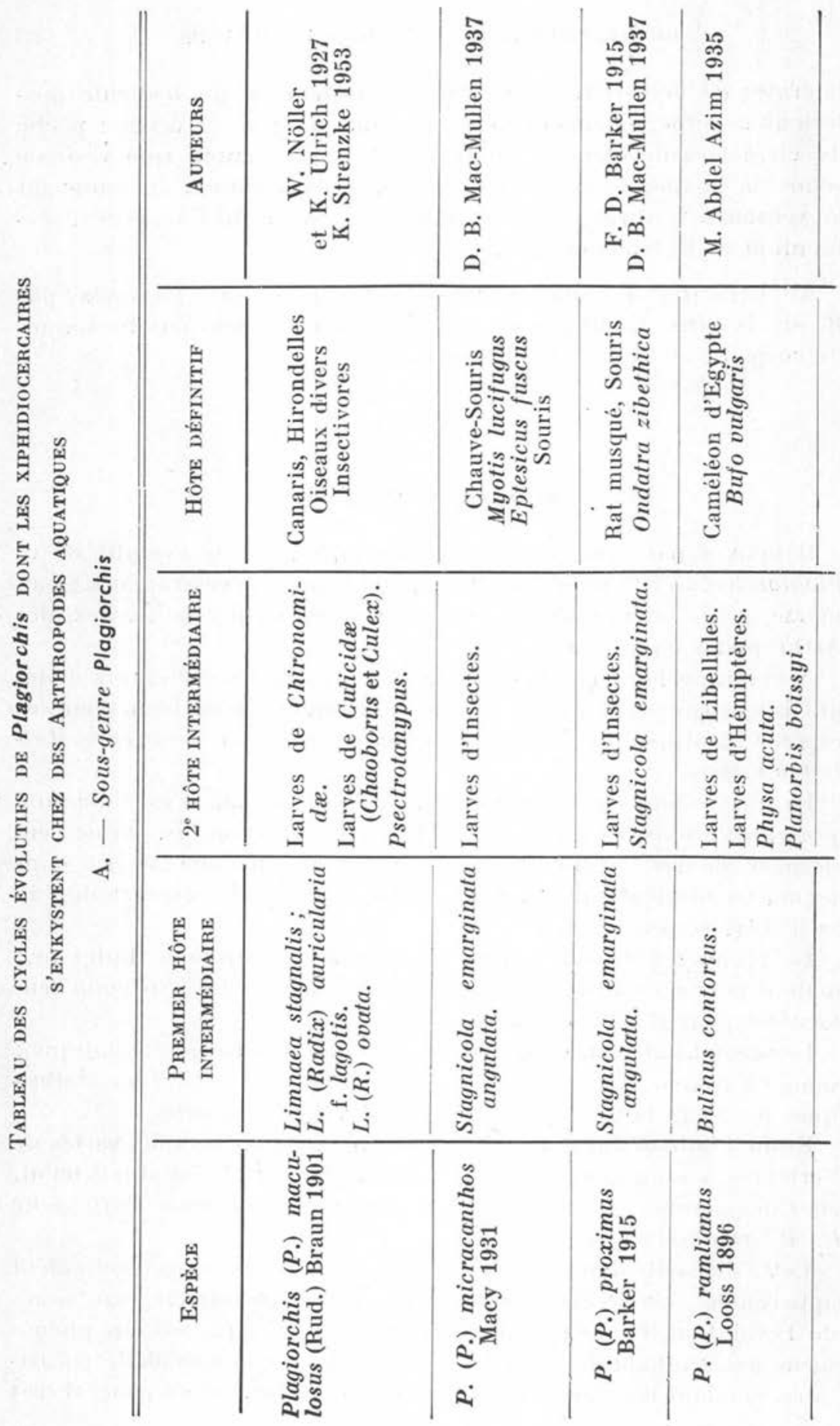




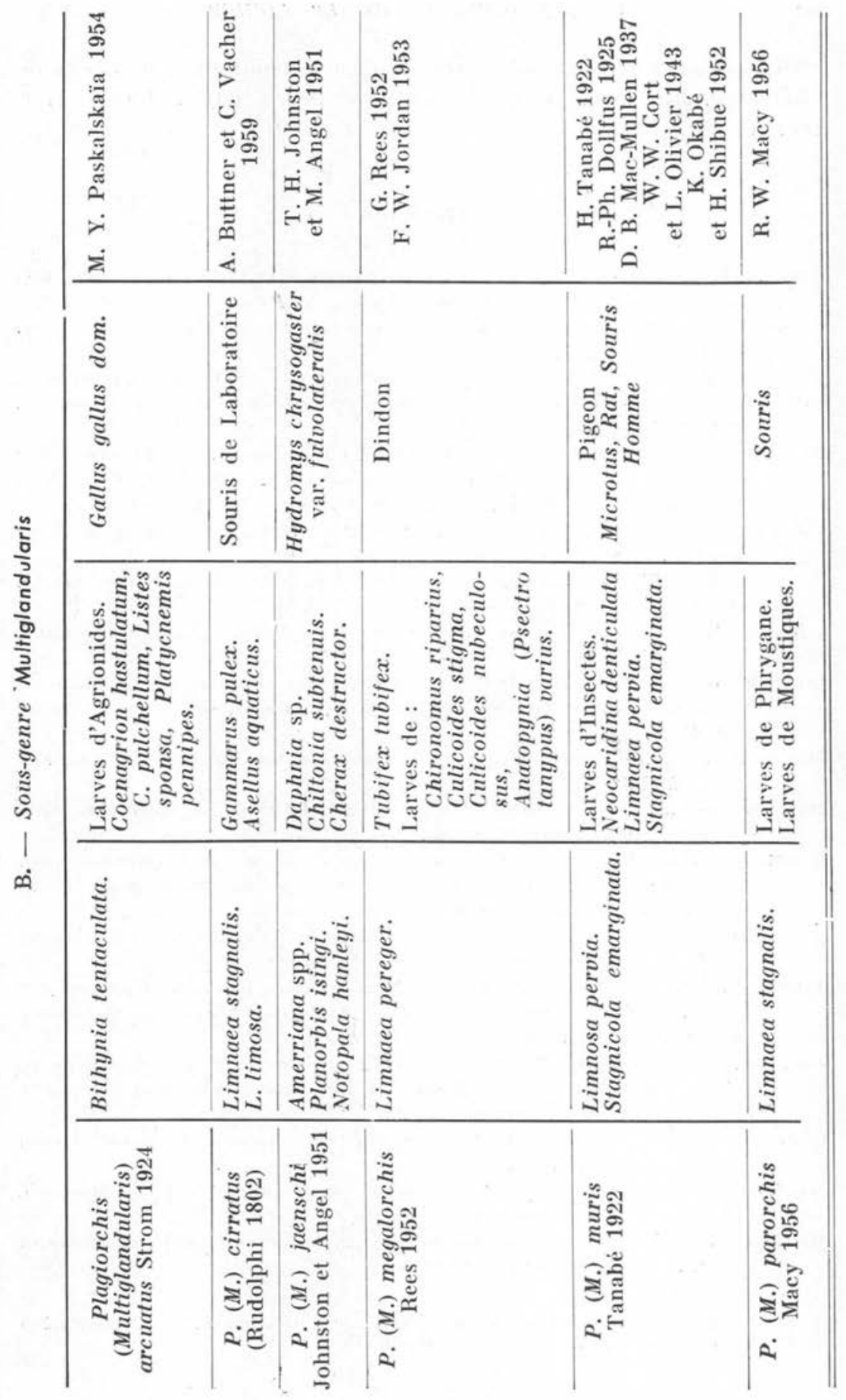


stricte. Elle n'a pas lieu de surprendre cependant, en raison de l'hétérogénéité déjà constatée dans le genre par différents auteurs (1).

\section{BiBLIOGRAPHIE}

Azıм (M. A.), 1935. - On the life story of Lepoderma ramlianus, Looss 1896 , and its development from a Xiphidiocercaria. Jl Parasit., XXI, p. 365-368.

BARKER (F. D.), 1915. - Parasites of the American muskrat (Fiber zibethicus). Jl Parasit., I, p. 192.

Dawes (Ben), 1946. - The Trematoda. Cambridge, Univ. Press, p. 321, p. 451-453.

Buttner (A.), 1950. - La progénèse chez les Trématodes digénétiques. Thèse Fac. Sci. Paris, p. 164, fig. 59 B.

Butroner (A.) et VACHer (C.), 1959. - Evolution d'un Plagiorchis s'enkystant chez Gammarus pulex L. et identifié à Plagiorchis cirratus (Rud. 1802) (Trematoda : Plagiorchiidre). C.R. Séance Soc. Biol., CLIII, n 11, p. 1712.

Cont (W. W.), 1914. - Larval Trematodes from North American fresh-water snails. Jl Parasit., I, p. 65-84.

Cont (W. W.), 1915. - Some North American larval Trematodes. Illinois biol. Monographs, I, 4, p. 56-57.

Cont (W. W.), 1919. - The excretory system of a stylet cercaria. Univ. Calif. Pub. Zool., XIX, 7, p. 275-281.

Cont (W. W.) et Olivier (L.), 1943. - The development of the larval stages of Plagiorchis muris Tanabé 1922 in the first intermediate host. Jl Paras., 29, 81-99.

Dubors (G.), 1929. - Les cercaires de la région de Neuchâtel. Thèse Fac. Scí. Neuchâtel.

Dollfus (R.-Ph.), 1925. - Distomiens parasites de Muride du genre Mus. Ann. Parasit. hum. comp., III, p. 188.

Erнardova (B.), 1955. - [Trematodes from Microtus aconomus mehelyi (Rodentia, Muridæ)]. Ceskoslovenska Parasitologie, II, p. 38-40 [Résumés en russe et en allemand, p. 39-40].

Grrmain (L.), 1930. - Faune de France, XXI. Mollusques terrestres et fluviatiles, Paris, Lechevalier édit.

Jordan (F. T. W.), 1953. - Intestinal infection of turkey poults with Plagiorchis (Multiglandularis) megalorchis Rees 1952, and an experimental study of its life-cycle. Jl Helminth., XXIII, 1/2, p. 75-80.

Johnston (T. H.) et ANGel (M.), 1951. - The life history of Plagiorchis jaenschi, a new Trematode from the Australian water-Rat. Trans. Roy. Soc. South Austral., LXXIV, p. 1, 49-58.

Lüне (M.), 1909. - Parasitische Plattwürmer, Trematodes. Süsswasser Fauna Deutschlands, Heft, 17.

MAC Coy (O. R.), 1928. - Life-story studies on Trematodes from Missouri. Jl Parasit., XIV, p. 207-228.

Mac Mullen (D. B.), 1937. - An experimental infection of Plagiorchis muris in man. Jl Parasit., XXII, p. 113-115.

(1) Voir à ce sujet, faisant suite à cet article : \& Description et identification de $P$. (M.) cirratus (Rud., 1902) 》 par R.-Ph. Dollfus, p. 
Mac Mullex (D. B.), 1937. - The life histories of three Trematodes, parasitic in Birds and Mammals, belonging to the genus Plagiorchis. Jl Parasit., XXIII, 3, p. 235-242.

MACY (R. W.), 1931. - New bat Trematodes of the genera Plagiorchis, Limatulum, and Dicrocalium. Il Parasit., XVIII, p. 28-33.

Niscy (R. W.), 1956. - The life cycle of Plagiorchis parorchis, n. sp. (Trematoda: Plagiorchiida). Il Parasit., XVII, 4, sect. 2, p. 28.

Nöller et Ullarich (K.), 1927. - Die Entwicklung einer Plagiorchis Art. Silzungsterich der Gesellschaft naturf.

OKAвÉ (K.) et ShibUe (H.), 1952. - A new second intermediaire host, Neocaridina denticulata for Plagiorchis muris (Tanabé) : Plagiorchiidæ. Japanese Jl Med. Sci. Biol., V, 5, p. 257-258.

P'askalkaya (M. Y.), 1954. - [The life cycle of the Trematoda Plagiorchis arcuatus, parasitic in the oviduct and bursa Fabricii of chickens]. Dokladi akademii Nauk S.S.S.R., XCVII, 3, p. 561-563 (en russe).

Paskalkaya (M. Y.), 1955. - [A new disease of hensplagiorchiasis]. Veterinariga, XXXIV, 4, p. 37-39 (en russe).

REEs (G.), 1952. - The structure of the adult and larval stages of Plagiorchis (Multiglandularis) megalorchis n. nom. from the turkey and an experimental demonstration of the life-history. Porasitology, XVII, 1-2, p. 92114.

Sewell (R. B. Seymour), 1922. - Cercaria indica. Ind. Jl med. Res., X, suppl. nr., p. 223-224.

STRENzKe (K.), 1952. - Der Wirtswechsel von Plagiorchis maculosus. Zeitschrift für Parasitenkunde, XV, 5, p. 369-391.

Strenzke (K.), 1953. - Zuckmücken als Zwischenwirte für Sangwürmer. Mikrokosmos, XLVIII, 8, p. 169-174.

TaNaBk (H.), 1922. - A contribution to the knowledge of the developmental cycle of digenetic Trematodes. On a new spccies of Trematode Lepoderma muris n. sp. Okayoma Igakkai Zasshi, $\mathrm{n}^{\circ} 385$, febr. 1922, p. 47-58, fig. 1-7 (en japonais).

Wesenerg-Lund (C.), 1931. - Contribution to the development of the Trematoda Digenea. Copenhague, p. 74-76.

Yamagut (Satyu), 1933. - Studies of the Helminth fauna of Japan. Part I. Trematodes of Birds, Reptiles and Mammals. Jap. Jl Zool., V, p. 1-134.

Yamaguti (Satyu), 1958. - Systema Helminthum, I, 1, p. 804-807, New-YorkLondres.

YAMAShita (J.), 1953. - [Studies on the daily fluctuation of the egg production of the rat Trematode Plagiorchis muris (Tanabé). I. On a case in the white mouse supplied with the crashed-rice or oat]. Memoirs of the Faculty of Agriculture, Hokkaido University, I, 3, p. 305-308 (en japonais, résumé en anglais p. 308). 